\title{
Momentum flux density, kinetic energy density and their fluctuations for one-dimensional confined gases of non-interacting fermions
}

\author{
Anna Minguzzi, Patrizia Vignolo and M. P. Tosi \\ Istituto Nazionale di Fisica della Materia and Classe di Scienze, Scuola Normale Superiore, Piazza dei Cavalieri 7, I-56126 \\ Pisa, Italy
}

\begin{abstract}
We present a Green's function method for the evaluation of the particle density profile and of the higher moments of the one-body density matrix in a mesoscopic system of $N$ Fermi particles moving independently in a linear potential. The usefulness of the method is illustrated by applications to a Fermi gas confined in a harmonic potential well, for which we evaluate the momentum flux and kinetic energy densities as well as their quantal mean-square fluctuations. We also study some properties of the kinetic energy functional $E_{k i n}[n(x)]$ in the same system. Whereas a local approximation to the kinetic energy density yields a multi-valued function, an exact single-valued relationship between the density derivative of $E_{k i n}[n(x)]$ and the particle density $n(x)$ is demonstrated and evaluated for various values of the number of particles in the system.
\end{abstract}

PACS numbers: 03.75.Fi, 05.30.Fk, 31.15.Ew

\section{INTRODUCTION}

The study of ultracold gases of fermionic alkali atoms is being pursued experimentally [1] by using basically the same trapping and cooling techniques which have led in 1995 to the achievement of Bose-Einstein condensation in gases of bosonic isotopes 24 . The main focus of the experimental effort is towards the realization of novel superfluids from pairing between fermions in different hyperfine states. However, attention has also been given to dilute Fermi gases in a fully spin-polarized state under magnetic confinement. Such a system presents special motives of fundamental interest from the fact that the Pauli principle suppresses the interactions in the $s$-wave scattering channel and thereby essentially isolates the kinetic energy functional which is invoked in the density functional theory of inhomogeneous fluids [5].

A quasi-one dimensional Fermi gas can be realized in the experiments [1] inside a very anisotropic magnetic trap, producing approximately harmonic confinement which is very tight in two directions. The particle and kinetic energy densities of the corresponding one-dimensional (1D) model of non-interacting fermions in a harmonic potential well have been evaluated exactly up to mesoscopic numbers of particles [6]. The shell structure in the particle density which was noticed in earlier studies of 3D Fermi gases [7,8, is greatly enhanced in lower dimensionality (see also Ref. [9]). As a further motive of interest, the 1D model of noninteracting fermions has the same particle density profile and thermodynamic properties as the 1D model of point-like bosons with hard core interactions 10 . The system of interacting bosons in $1 \mathrm{D}$ is attracting attention due to its rich phase diagram [13].

The particle and kinetic energy densities are the zero-th moment and a second moment of the one-body density matrix. In our earlier study [6] we have outlined a general method by which one may calculate the successive moments of the density matrix in a system of $N$ Fermi particles moving independently in a 1D potential. In the fully quantum regime alternative definitions can be given for the higher-order moments, depending on how the operators are symmetrized [14]. In particular, two different second-order moments can be defined for an inhomogeneous gas under confinement, yielding two different physical quantities which are the kinetic energy density and the momentum flux density. The method here proposed is suitable for the higher-order moments in any of their forms. It exploits the definition of the Green's function operator $\hat{G}(x)$ in coordinate space and allows one to use the numerical methods already developed in solid state physics [15 17 to evaluate the density of single-particle states from the Green's function in the energy domain.

In this paper we give a detailed account of the general method in Sec. II and some new applications to the case of harmonic confinement in the following Sections. In Sec. III we contrast the kinetic energy density with the momentum flux density in the presence of inhomogeneity. In Sec. IV we assess the quantal (zero-temperature) fluctuations in the kinetic energy and momentum flux densities by means of a calculation of fourth moments of the one-body density matrix. Properties of the kinetic energy functional are discussed in Sec. $\mathrm{V}$ in the context of density functional theory. An exact local-density method exists for the system of present interest, as shown in a different approach by Lawes and March [18], and is explicitely exhibited here for various values of the number of Fermi particles. Finally, in Sec. VI we summarize our main conclusions. 


\section{THE METHOD}

We consider a system of $N$ non-interacting Fermi particles in one spatial dimension subjected to an external confinement given by a real potential $V_{\text {ext }}(x)$. We may take the eigenfunctions $\psi_{i}(x)$ as real [19. The one-body density matrix at zero temperature is

$$
\rho\left(x, x_{1}\right)=\sum_{i=1}^{N} \psi_{i}(x) \psi_{i}\left(x_{1}\right)
$$

Its zero-th moment is the equilibrium density profile $n(x)$, and its odd moments are zero due to the reality of the wavefunctions. For the even moments of higher order several definitions have been considered in the literature (for a review see e.g. Ziff et al. [14), among which we shall focus on the following:

$$
P_{n}(x)=(-i)^{n}\left[\frac{\partial^{n}}{\partial x_{1}^{n}} \rho\left(x, x_{1}\right)\right]_{x_{1}=x}
$$

and

$$
S_{n}(x)=(-i)^{n}\left[\frac{\partial^{n}}{\partial r^{n}} \rho(x+r / 2, x-r / 2)\right]_{r=0}
$$

(we have set $\hbar=1$ and $m=1$ ). For $n \geq 2$ these definitions give rise to different equilibrium properties in an inhomogeneous system, although their semiclassical limits and their integrals coincide. In the specific case of $n=2$ Eqs. (2) and (3) give twice the kinetic energy density and the momentum flux density, respectively: these will be discussed in Sec. III.

The method developed in Ref. [6] is here extended to evaluate both $P_{n}(x)$ and $S_{n}(x)$. For this purpose we write Eqs. (2) and (3) in terms of the Green's function $\hat{G}(x)=(x-\hat{x}+i \varepsilon)^{-1}$ in coordinate space as

$$
P_{n}(x)=-\frac{1}{\pi} \lim _{\varepsilon \rightarrow 0^{+}} \operatorname{Im} \sum_{i=1}^{N}\left\langle\psi_{i}\left|\hat{G}(x) \hat{p}^{n}\right| \psi_{i}\right\rangle
$$

and

$$
S_{n}(x)=-\frac{1}{2^{n} \pi} \sum_{\sigma=0}^{n}\left(\begin{array}{c}
n \\
\sigma
\end{array}\right) \lim _{\varepsilon \rightarrow 0^{+}} \operatorname{Im} \sum_{i=1}^{N}\left\langle\psi_{i}\left|\hat{p}^{\sigma} \hat{G}(x) \hat{p}^{n-\sigma}\right| \psi_{i}\right\rangle .
$$

Here, $\left|\psi_{i}\right\rangle$ are the eigenstates of the Hamiltonian in the coordinate representation and $\hat{p}$ is the momentum operator. Equation (4) is easily proved by making use of the expression of $\hat{G}(x)$ in the coordinate representation:

$$
\begin{aligned}
P_{n}(x) & =-\frac{(-i)^{n}}{\pi} \lim _{\varepsilon \rightarrow 0^{+}} \operatorname{Im} \sum_{i=1}^{N} \int \mathrm{d} x_{i} \psi_{i}\left(x_{i}\right) \frac{1}{x-x_{i}+i \varepsilon} \frac{\partial^{n}}{\partial x_{i}^{n}} \psi_{i}\left(x_{i}\right) \\
& =(-i)^{n} \sum_{i=1}^{N} \int \mathrm{d} x_{i} \psi_{i}\left(x_{i}\right) \delta\left(x-x_{i}\right) \frac{\partial^{n}}{\partial x_{i}^{n}} \psi_{i}\left(x_{i}\right),
\end{aligned}
$$

where $x_{i}$ are the eigenvalues of the position operator $\hat{x}$. Equation (5) can be proved in a similar fashion.

The momentum distribution $n(p)$ can be analogously written in terms of the trace of the Green's function $\hat{G}(p)=$ $(p-\hat{p}+i \varepsilon)^{-1}$ in momentum space, taken on the eigenvectors $\left|\phi_{i}\right\rangle$ of the Hamiltonian in the momentum representation:

$$
n(p)=\sum_{i=1}^{N} \int \mathrm{d} p_{i}\left|\phi_{i}\left(p_{i}\right)\right|^{2} \delta\left(p-p_{i}\right)
$$

or

$$
n(p)=-\frac{1}{\pi} \lim _{\varepsilon \rightarrow 0^{+}} \operatorname{Im} \sum_{i=1}^{N}\left\langle\phi_{i}|\hat{G}(p)| \phi_{i}\right\rangle
$$


where $p_{i}$ are the eigenvalues of the momentum operator.

To evaluate the Green's functions in the specific case of harmonic confinement, we make use of the representation for the position and the momentum operators in the basis of the eigenstates of the harmonic oscillator, i.e. $\hat{x}=\left(a+a^{\dagger}\right) / \sqrt{2}$ and $\hat{p}=i\left(a^{\dagger}-a\right) / \sqrt{2}$ with $a\left|\psi_{i}\right\rangle=\sqrt{i-1}\left|\psi_{i-1}\right\rangle$ and $a^{\dagger}\left|\psi_{i}\right\rangle=\sqrt{i}\left|\psi_{i+1}\right\rangle$ (we have set the harmonic oscillator frequency $\omega=1$ ). Explicitly, the representation of $\hat{x}$ in matrix form is given by

$$
\hat{x}=\left(\begin{array}{ccccccc}
0 & 1 / \sqrt{2} & & & & & \\
1 / \sqrt{2} & 0 & 1 & & & & \\
& 1 & 0 & \sqrt{3 / 2} & & & \\
& & \sqrt{3 / 2} & 0 & \sqrt{2} & & \\
& & & \sqrt{2} & 0 & \sqrt{5 / 2} & \\
& & & & \ddots & \ddots & \ddots
\end{array}\right)
$$

and similarly for $\hat{p}$. The various moments of the one-body density matrix and the momentum distribution are then evaluated as the trace of the operators $\hat{p}^{\sigma} \hat{G}(x) \hat{p}^{n-\sigma}$ and of $\hat{G}(p)$ on the first $N$ states $\left(\operatorname{Tr}_{N}\right)$. The trace is calculated by the general method given below. The quantities $n(x)$ and $n(p)$ could also be calculated from a suitably modified Kirkman-Pendry relation [20]17], as is shown in Appendix A.

\section{A. Expression for the evaluation of the trace}

The partial trace $\operatorname{Tr}_{N}$ of a generic matrix $Q$ is related to the determinant of the inverse matrix $Q^{-1}$ by

$$
\operatorname{Tr}_{N} Q=\partial\left[\ln \operatorname{det}\left(Q^{-1}+\lambda \mathbb{I}_{N}\right)\right] /\left.\partial \lambda\right|_{\lambda=0} .
$$

This relation is demonstrated as follows:

$$
\begin{array}{r}
\partial\left[\ln \operatorname{det}\left(Q^{-1}+\lambda \mathbb{I}_{N}\right)\right] /\left.\partial \lambda\right|_{\lambda=0}=\partial\left[\operatorname{Tr} \ln \left(Q^{-1}+\lambda \mathbb{I}_{N}\right)\right] /\left.\partial \lambda\right|_{\lambda=0} \\
=\operatorname{Tr}\left[\left(Q^{-1}+\lambda \mathbb{I}_{N}\right)^{-1} \mathbb{I}_{N}\right]_{\lambda=0}=\operatorname{Tr}\left(Q \mathbb{I}_{N}\right)=\operatorname{Tr}_{N} Q .
\end{array}
$$

Here we take $Q=\hat{p}^{\sigma} \hat{G}(x) \hat{p}^{n-\sigma}$. In the case of harmonic confinement this matrix is the product of a $(2 n+1)$-diagonal matrix and of the inverse of a tridiagonal matrix. Exploiting Eq. (10) we can write

$$
P_{n}(x)=-\frac{1}{\pi} \lim _{\varepsilon \rightarrow 0^{+}} \operatorname{Im} \frac{\partial}{\partial \lambda}\left[\ln \operatorname{det}\left(x+i \varepsilon-\hat{K}^{n, 0}\right)\right]_{\lambda=0},
$$

and

$$
S_{n}(x)=-\frac{1}{2^{n} \pi} \sum_{\sigma=0}^{n}\left(\begin{array}{c}
n \\
\sigma
\end{array}\right) \lim _{\varepsilon \rightarrow 0^{+}} \operatorname{Im} \frac{\partial}{\partial \lambda}\left[\ln \operatorname{det}\left(x+i \varepsilon-\hat{K}^{n, \sigma}\right)\right]_{\lambda=0}
$$

where $\hat{K}^{n, \sigma}=\hat{x}-\hat{p}^{n-\sigma} \lambda \mathbb{I}_{N} \hat{p}^{\sigma}$. We have thus reduced the problem to the evaluation of the determinant of the matrix $\left(x+i \varepsilon-\hat{K}^{n, \sigma}\right)$, which is tridiagonal in the tail and whose first $N$ rows have only a few non-vanishing elements for low values of $n$.

If we make a partition in blocks of dimension $n \times n$ for the first part of the matrix, the whole operator can be written as

$$
\hat{K}^{n, \sigma}=\lim _{M \rightarrow \infty} \hat{K}_{M}^{n, \sigma}=\lim _{M \rightarrow \infty}\left(\begin{array}{cccccc}
\mathcal{A}_{1} & \mathcal{B}_{1,2} & & & & \\
\mathcal{B}_{2,1} & \mathcal{A}_{2} & \mathcal{B}_{2,3} & & & \\
& \mathcal{B}_{3,2} & \ddots & \mathcal{B}_{3,4} & & \\
& & \ddots & \ddots & \ddots & \\
& & & \ddots & \ddots & \mathcal{B}_{M-1, M} \\
& & & & \mathcal{B}_{M, M-1} & \mathcal{A}_{M}
\end{array}\right) .
$$

Here, we have introduced the truncated matrix $\hat{K}_{M}^{n, \sigma}$, which will be used below in actual numerical calculations. 


\section{B. The renormalization procedure}

The determinant of the matrix $\left(x+i \varepsilon-\hat{K}^{n, \sigma}\right)$ with a tridiagonal representation such as that in Eq. (14) can be factorized into a product of determinants of matrices having the same dimension as that of the blocks of the partition [17].

As a first step, it is easy to show that if we partition the matrix $\hat{K}_{M}^{n, \sigma}$ into four blocks, thereby defining $\tilde{\mathcal{B}}_{i, j}$ an $\hat{K}_{i}^{n, \sigma}$ from

$$
\hat{K}_{M}^{n, \sigma}=\left(\begin{array}{cc}
\hat{K}_{M-1}^{n, \sigma} & \tilde{\mathcal{B}}_{M-1, M} \\
\tilde{\mathcal{B}}_{M, M-1} & \mathcal{A}_{M}
\end{array}\right)
$$

we can write

$$
\operatorname{det}\left(x+i \varepsilon-\hat{K}_{M}^{n, \sigma}\right)=\operatorname{det}\left(x+i \varepsilon-\hat{K}_{M-1}^{n, \sigma}\right) \cdot \operatorname{det}\left(x+i \varepsilon-\mathcal{A}_{M}-\tilde{\mathcal{B}}_{M, M-1}\left(x+i \varepsilon-\hat{K}_{M-1}^{n, \sigma}\right)^{-1} \tilde{\mathcal{B}}_{M-1, M}\right) .
$$

Applying recursively this procedure and taking the limit $M \rightarrow \infty$ we obtain

$$
\operatorname{det}\left(x+i \varepsilon-\hat{K}^{n, \sigma}\right)=\operatorname{det}\left(x+i \varepsilon-\mathcal{A}_{1}\right) \cdot \lim _{M \rightarrow \infty} \prod_{j=2}^{M} \operatorname{det}\left(x+i \varepsilon-\mathcal{A}_{j}-\tilde{\mathcal{B}}_{j, j-1}\left(x+i \varepsilon-\hat{K}_{j-1}^{n, \sigma}\right)^{-1} \tilde{\mathcal{B}}_{j-1, j}\right) .
$$

It is now important to notice that, owing to the particular form of the matrices $\tilde{\mathcal{B}}_{j-1, j}$ and $\tilde{\mathcal{B}}_{j, j-1}$, it is not necessary to explicitly invert the matrices $\left(x+i \varepsilon-\hat{K}_{j-1}^{n, \sigma}\right)$. Rather, we may use a renormalization procedure for the operator $\hat{K}_{j-1}^{n, \sigma}$ to further simplify the expression (17) and to calculate the inverse of matrices having dimension at most equal to $n \times n$. Renormalization is based on the idea that if one only needs to describe a given subspace of the whole Hilbert space of the system, one can define an effective operator acting in the subspace and taking into account the contribution from the rest of the system. Renormalization allows us to write

$$
\operatorname{det}\left(x+i \varepsilon-\hat{K}^{n, \sigma}\right)=\prod_{j=1}^{\infty} \operatorname{det}\left(x+i \varepsilon-\tilde{\mathcal{A}}_{j}\right)
$$

where $\tilde{\mathcal{A}}_{1}=\mathcal{A}_{1}$ and

$$
\tilde{\mathcal{A}}_{j}=\mathcal{A}_{j}+\mathcal{B}_{j, j-1}\left(x+i \varepsilon-\tilde{\mathcal{A}}_{j-1}\right)^{-1} \mathcal{B}_{j-1, j}
$$

for $j>1$. The $\mathcal{B}_{j, j+1}$ have been introduced in Eq. (14). In the specific case of $n=2$ and $\sigma=0$ Eq. (18) yields back the expression obtained in Ref. [6].

Operatively, we have studied the convergence of the function $\operatorname{det}\left(x+i \varepsilon-\hat{K}_{M}^{n, \sigma}\right)$ on increasing $M$. We have performed our calculations up to $n=4$ and $N=1000$ fermions with $M \sim 10^{7}$ and $\varepsilon=0.001$.

\section{KINETIC ENERGY AND MOMENTUM FLUX DENSITIES}

The second moments $P_{2}(x)$ and $S_{2}(x)$ of the one-body density matrix from Eqs. (2) and (3) have different physical meanings and show different behaviours. The function $P_{2}(x)=\sum_{i}^{N}\left\langle\psi_{i}\left|\delta\left(x-x_{i}\right) \hat{p}^{2}\right| \psi_{i}\right\rangle$ is twice the kinetic energy density $T(x)$ [18] and for harmonic confinement has already been evaluated in Ref. [6] together with the equilibrium density profile. This quantity is of main interest in the context of Density Functional Theory, as will be discussed in Sec. V. On the other hand, $S_{2}(x)=(1 / 2) \sum_{i}^{N}\left\langle\psi_{i}\left|\delta\left(x-x_{i}\right) \hat{p}^{2}+\hat{p} \delta\left(x-x_{i}\right) \hat{p}\right| \psi_{i}\right\rangle$ is the momentum flux density $\Pi(x)$ which enters the equations of generalized hydrodynamics [21]. In 3D fluids this quantity becomes a tensor, known as the kinetic stress tensor.

The equation of motion of the particle density $n(x, t)$ for non-interacting fermions in $1 \mathrm{D}$ is

$$
\frac{\partial^{2}}{\partial t^{2}} n(x, t)=\frac{\partial^{2}}{\partial x^{2}} \Pi(x, t)+\frac{\partial}{\partial x}\left[n(x, t) \frac{\partial}{\partial x} V_{e x t}(x, t)\right] .
$$

At equilibrium in a static potential we obtain from Eq. (20) an exact relation between the momentum flux density and the density profile,

$$
\frac{d}{d x} \Pi(x)=-n(x) \frac{d}{d x} V_{e x t}(x)+\text { const }
$$


The integration constant can be fixed by imposing suitable boundary conditions for $x \rightarrow \pm \infty$.

Another exact relation can be derived by exploiting Eqs. (11)-(3) together with the definition of $n(x)$ as the zero-th moment of the density matrix. This reads:

$$
\Pi(x)=2 T(x)+\frac{1}{4} \frac{d^{2}}{d x^{2}} n(x) .
$$

Equation (22) exhibits the relation which exists between the momentum flux density and the kinetic pressure $P(x)=$ $2 T(x)$ in the inhomogeneuos system. Evidently, in the homogeneous limit the momentum flux density and the kinetic pressure coincide - and therefore they also coincide within a local-density description for the confined gas. They instead differ within a quantum-mechanical description of the the inhomogeneous system, when one keeps exactly into account the role of confinement. This difference is readily illustrated in the fully quantum case of a single fermion in an harmonic oscillator potential, where we have

$$
\Pi(x)=T(x)+V(x)
$$

with $V(x)=n(x) V_{\text {ext }}(x)$ being the potential energy density associated with the confinement.

For the case of harmonic confinement we have employed the method described in Sec. II to evaluate the exact profiles for both $T(x)$ and $\Pi(x)$ at various numbers of particles. Figure 1 shows these results for $N=4,12$ and 24 fermions. While $T(x)$ shows $N$ oscillations and negative tails [6], $\Pi(x)$ is everywhere positive and its shell structure is visible only in its first derivative (see inset in Fig. 1).

\section{QUANTUM FLUCTUATIONS OF THE KINETIC ENERGY AND MOMENTUM FLUX DENSITIES}

From the fourth moments $S_{4}(x)$ and $P_{4}(x)$ of the density matrix we can evaluate the local fluctuations of the momentum flux and kinetic energy densities. For the former the fluctuations are most conveniently introduced through the Wigner distribution function,

$$
f_{W}(p, x)=\int d r e^{i p r} \rho(x+r / 2, x-r / 2) .
$$

In this representation the relevant moments are given by $n(x)=\sum_{p} f_{W}(p, x)$ and by $\Pi(x)=\sum_{p} p^{2} f_{W}(p, x) \equiv$ $n(x)\left\langle p^{2}\right\rangle$. The mean square fluctuation of the momentum flux density is therefore given by

$$
\Delta \Pi(x)=\sum_{p}\left(p^{2}-\left\langle p^{2}\right\rangle\right)^{2} f_{W}(p, x)=S_{4}(x)-\Pi^{2}(x) / n(x),
$$

where $S_{4}(x)$ is as defined in Eq. (3). Equation (25) in the coordinate representation for the density matrix can be rewritten as

$$
\Delta \Pi(x)=\left.\left[-\frac{\partial^{2}}{\partial r^{2}}-\frac{\Pi(x)}{n(x)}\right]^{2} \rho(x+r / 2, x-r / 2)\right|_{r=0} .
$$

The fluctuations of the kinetic energy density are obtained in a similar fashion as

$$
\begin{aligned}
\Delta T(x) & =\left.\left[-\frac{1}{2} \frac{\partial^{2}}{\partial x_{1}^{2}}-\frac{T\left(x_{1}\right)}{n\left(x_{1}\right)}\right]^{2} \rho\left(x, x_{1}\right)\right|_{x_{1}=x} \\
& =\frac{1}{4} P_{4}(x)-\frac{T^{2}(x)}{n(x)}+\frac{1}{2} \frac{d}{d x}\left[n(x) \frac{d}{d x}\left(\frac{T(x)}{n(x)}\right)\right],
\end{aligned}
$$

with $P_{4}(x)$ as defined in Eq. (2).

For harmonic confinement in the local density approximation (LDA) we have $n_{L D A}(x)=\left(2 N-x^{2}\right)^{1 / 2} / \pi, \Pi_{L D A}(x)=$ $2 T_{L D A}(x)=\left(2 N-x^{2}\right)^{3 / 2} / 3 \pi$ and $S_{4}^{L D A}(x)=P_{4}^{L D A}(x)=\left(2 N-x^{2}\right)^{5 / 2} / 5 \pi$. It is straightforward to show from these expressions that the integrated mean square fluctuations, divided by the square of the corresponding integrated quantity, scale as $1 / N$. The same scaling is found in the exact numerical results at large $N$.

We have used the Green's function method described in Sec. II to evaluate the exact profiles for both $\Delta \Pi(x)$ and $\Delta T(x)$ in harmonic confinement, as shown in Fig. 2 for $N=4,12$ and 24 fermions. The positions of the $N-1$ maxima in $\Delta T(x)$ are located in correspondence to the minima of the kinetic energy density in Fig. 1 (a), and again negative tails are present at the boundaries. In the case of $N=2$ we have tested our numerical results against the analytic expression given for $\Delta T(x)$ by low-order Hermite polynomials, the result being shown in Fig. 3. Evidently, we have obtained in this case a very accurate numerical evaluation of up to the fourth moment of the density matrix. 


\section{PROPERTIES OF THE KINETIC ENERGY FUNCTIONAL}

We have seen that the second moment $P_{2}(x)$ of the one-body density matrix yields the kinetic energy density $T(x)=P_{2}(x) / 2$. The total kinetic energy is obtained by integration over the spatial coordinate and in the spirit of Density-Functional Theory (DFT) [5] can be viewed as a functional of the particle density $n(x)$ :

$$
E_{k i n}[n(x)]=\int d x T(x)=\int d x \hat{T}[n(x)] .
$$

Here we have introduced an operator $\hat{T}$ which acts on the density profile to yield

$$
\hat{T}[n(x)]=T(x) .
$$

A main point of interest for DFT is to assess the properties of the unknown operator $\hat{T}$.

Knowing exactly the function $T(x)$ the specific case of 1D harmonic confinement, we may try to construct a function $\tilde{T}(n)=T(x(n))$ in a local density approach, with $x(n)$ being given by inversion of the density profile. The inversion has to be performed locally, since the exact density profile is not globally invertible owing to its shell structure. The resulting $\tilde{T}(n)$, as reported in Fig 1 , is a multi-valued function around the values of the particle density corresponding to each local maximum in the profile. While this shows that a local-density approach cannot characterize the operator $\hat{T}$, further progress can be made by studying the derivative of $\tilde{T}(n)$ with respect to the density, which is the local chemical potential $\tilde{\mu}_{l o c}(n)=d \tilde{T}(n) / d n$.

We show here that for the specific case of a $1 \mathrm{D}$ harmonic confinement the inverse $\tilde{n}\left(\mu_{l o c}\right)$ of the function $\tilde{\mu}_{l o c}(n)$ is a single-valued function. This is most simply seen by inverting the Euler equation

$$
\frac{\delta E_{k i n}[n(x)]}{\delta n(x)}=\mu-V_{e x t}(x) \equiv \mu_{l o c}(x)
$$

in the domain $x \geq 0$ and by substituting its value into the density profile $n(x)$ to obtain

$$
\tilde{n}\left(\mu_{l o c}\right)=n\left(x\left(\mu_{l o c}\right)\right) .
$$

Here, $x\left(\mu_{l o c}\right)$ is the inverse of the function $\mu_{l o c}(x)$. Because of the symmetry $x \leftrightarrow-x$ for the harmonic potential, the region $x<0$ is not needed to obtain $\tilde{n}\left(\mu_{l o c}\right)$.

An analytic calculation for $\tilde{n}\left(\mu_{l o c}\right)$ has been reported by Lawes and March [18] for $N=1$ and 2. The numerical method described in Sect. II allows us to easily compute the density profile for numbers of particles up to $N=1000$. By combining these results for $n(x)$ with the analytic expression for $x\left(\mu_{l o c}\right)$, we have obtained the function $\tilde{n}\left(\mu_{l o c}\right)$ which is shown in Fig. 5 for various values of $N$. At variance from the LDA prediction, the exact density profile is non-vanishing at negative values of $\mu_{l o c}$ and shows a number of oscillations (equal to $N / 2$ for even $N$ or to $(N-1) / 2$ for odd $N$ ), with decreasing amplitude as $N$ increases.

The property of single-valuedness of $\tilde{n}\left(\mu_{l o c}\right)$ is a characteristic of $1 \mathrm{D}$ harmonic confinement and can be understood to be a consequence of the local nature of the relation $\tilde{n}\left(\mu_{l o c}\right) v s . \mu_{l o c}$, which was already pointed out by Lawes and March [18]. Anharmonicity would be sufficient to make it invalid.

\section{SUMMARY AND CONCLUSIONS}

In summary, we have presented a general method for evaluating the $n$-th moments of the one-body density matrix (defined either through the derivatives with respect to the relative coordinate or through the derivatives with respect to the second position variable) for a system of confined non-interacting $1 \mathrm{D}$ fermions. We have applied this method to the case of harmonic confinement to calculate up to fourth moments.

Regarding second moments, the momentum flux density $\Pi(x)$ has been computed and compared with the kinetic energy density $T(x)$ at various numbers of particles. While $T(x)$ shows a prominent shell structure and negative tails, $\Pi(x)$ is everywhere positive and has a less marked shell structure, which can be evidenced in its first spatial derivative. The relation between these two different physical quantities has been elucidated by giving an exact relation between them and the equilibrium density profile of the fermion cloud. The momentum flux density determines the dynamical properties of the cloud, whereas the kinetic energy density is relevant in the context of Density Functional Theory.

We have also calculated the fourth moments of the density matrix in order to display the quantal fluctuations of the kinetic energy and momentum flux densities. Finally, we have evaluated the local relationship which exists between density profile and local chemical potential for fermion clouds in 1D harmonic confinement. This relation has allowed us to display an exact property of the DFT kinetic energy functional for a 1D Fermi gas up to mesoscopic values of the number of particles. 


\section{ACKNOWLEDGMENTS}

This work was partially supported by MURST through PRIN2000. One of us (A.M.) would like to thank Dr. F. Illuminati for useful discussions and for giving reference to the work of Ziff et al. 14 .

\section{APPENDIX A: DENSITY PROFILE FROM THE KIRKMAN-PENDRY RELATION}

We derive in this Appendix an expression alternative to Eq. (12) for the evaluation of the density profile in a system of $N$ fermions under 1D harmonic confinement. The trace of the Green's function $\hat{G}(x)$ over its first $\mathrm{N}$ states in Eq. (4) is obtained through an extension of the Kirkman-Pendry relation [20], already used for the density of states in a quasi-1D system 15,17 .

We define the Green's function

$$
\hat{\mathcal{G}}(\delta, x)=\frac{1}{x+\delta-\hat{\xi}(x)+i \varepsilon}
$$

where $\delta$ is an auxiliary continuous variable and $\hat{\xi}(x)$ is an effective position operator of dimension $N \times N$. This is defined by setting $[\hat{\xi}(x)]_{i, j}=[\hat{x}]_{i, j}$ if $(i, j) \neq(N, N)$ and $[\hat{\xi}(x)]_{N, N}=\tilde{x}_{N, N}(x)$, with

$$
\tilde{x}_{N, N}(x)=\frac{N / 2}{x+i \varepsilon-\frac{(N+1) / 2}{x+i \varepsilon-\ldots}} .
$$

The single term $\tilde{x}_{N, N}(x)$ contains the contribution of all the states which are not occupied by the fermions. Its asymptotic value is $\tilde{x}_{N, N}(x)=i \sqrt{2 N-x^{2}}$ for $N \rightarrow \infty$.

An expression for $\operatorname{Tr}_{N} \hat{G}(x)$ is obtained from the Green's function element $\mathcal{G}_{1, N}(\delta, x)$ between the first and the last occupied state by using the expression

$$
\begin{aligned}
\frac{\partial}{\partial \delta} \ln \mathcal{G}_{1, N}(\delta, x) & =\frac{\partial}{\partial \delta} \ln \frac{\prod_{i=1}^{N-1}[\hat{\xi}(x)]_{i, i+1}}{\operatorname{det}[x+\delta-\hat{\xi}(x)+i \varepsilon]}=\frac{\partial}{\partial \delta} \ln \frac{\prod_{i=1}^{N-1}[\hat{\xi}(x)]_{i, i+1}}{\prod_{i=1}^{N}\left[x+\delta-\xi_{i}(x)+i \varepsilon\right]} \\
& =\frac{\partial}{\partial \delta} \ln \prod_{i=1}^{N} \frac{1}{x+\delta-\xi_{i}(x)+i \varepsilon}=\sum_{i=1}^{N} \frac{-1}{x+\delta-\xi_{i}(x)+i \varepsilon}
\end{aligned}
$$

where $\xi_{i}(x)$ are the eigenvalues of the operator $\hat{\xi}(x)$. Therefore, the particle density is given by

$$
n(x)=-\frac{1}{\pi} \lim _{\varepsilon \rightarrow 0^{+}} \operatorname{Im}_{\operatorname{Tr}_{N}} \hat{G}(x)=\frac{1}{\pi} \lim _{\varepsilon \rightarrow 0^{+}} \operatorname{Im}\left[\frac{\partial}{\partial \delta} \ln \mathcal{G}_{1, N}(\delta, x)\right]_{\delta=0} .
$$

The matrix element $\mathcal{G}_{1, N}(\delta, x)$ can be calculated by a further renormalization 15 of $\hat{\xi}(x)$, without explicitly inverting the matrix $(x+\delta-\hat{\xi}(x)+i \varepsilon)$. As in the method presented in Sec. III, convergence is achieved by setting $M=10^{7}$ and $\varepsilon=0.001$.

The same procedure can be used to evaluate the momentum distribution as the trace of an $N \times N$ operator $\hat{\pi}(p)$, defined analogously to $\hat{\xi}(x)$. Due to the symmetry between the operators $\hat{x}$ and $\hat{p}$ in the harmonic oscillator Hamiltonian, we obtain essentially the same final expression as for of the particle density $n(x)$. Thus, the momentum distribution for $N$ fermions under 1D harmonic confinement shows $N$ oscillations.

The mapping between hard-core bosons and non-interacting fermions in 1D [10 allows us to evaluate the particle density profile for mesoscopic systems of strongly correlated bosons by the same method. However, the mapping is restricted to real-space properties and does not apply to the momentum distribution.

[1] B. DeMarco and D. S. Jin, Science 285, 1703 (1999). 
[2] M. H. Anderson, J. R. Ensher, M. R. Matthews, C. E. Wieman, and E. A. Cornell, Science 269, 198 (1995).

[3] K. B. Davis, M. O. Mewes, M. R. Andrews, N. J. van Druten, D. S. Durfee, D. M. Kurn, and W. Ketterle, Phys. Rev. Lett. 75, 3969 (1995).

[4] C. C. Bradley, C. A. Sackett, J. J. Tollet, and R. G. Hulet, Phys. Rev. Lett. 75, 1687 (1995); see also ibid. 79, 1170 (1997).

[5] S. Lundqvist and N. H. March, Theory of the Inhomogeneous Electron Gas (Plenum, New York, 1983); E. K. U. Gross, J. K. Dobson, and M. Petersilka, in Topics in Current Chemistry, ed. R. F. Nalewajski (Springer, Berlin, 1996$)$, p. 1.

[6] P. Vignolo, A. Minguzzi, and M. P. Tosi, Phys. Rev. Lett. 85, 2850 (2000). A misprint has occurred in the first line of Eq. (3), which should read $P(x)=-\left.\left(\hbar^{2} / m\right)\left(\partial^{2} / \partial x_{1}^{2}\right) \rho\left(x, x_{1}\right)\right|_{x_{1}=x}$. However, the second line of Eq. (3) for $P(x)$ and all the following calculations are correct.

[7] J. Schneider and H. Wallis, Phys. Rev. A 57, 1253 (1998).

[8] G. M. Bruun and K. Burnett, Phys. Rev. A 58, 2427 (1998).

[9] F. Gleisberg, W. Wonneberger, U. Schlöder and C. Zimmermann, Phys. Rev. A 62, 063602 (2000).

[10] M. D. Girardeau, Journ. Math. Phys. 1, 516 (1960).

[11] M. D. Girardeau and E. M. Wright, Phys. Rev. Lett. 84, 5239 (2000).

[12] E. B. Kolomeisky, T. J. Newman, J. P. Straley, and X. Qi, Phys. Rev. Lett. 85, 1146 (2000).

[13] D. S. Petrov, G. V. Shlyapnikov, and J. T. M. Walraven, cond-mat/0006339.

[14] R. M. Ziff, G. E. Uhlenbeck, and M. Kag, Phys. Rep. 32, 169 (1977).

[15] P. Vignolo, R. Farchioni, and G. Grosso, Phys. Rev. B 59, 16065 (1999).

[16] R. Farchioni, P. Vignolo, and G. Grosso, Phys. Rev. B 60, 15705 (1999).

[17] R. Farchioni, G. Grosso and P. Vignolo, Phys. Rev. B 62, 12565 (2000).

[18] G. P. Lawes and N. H. March, J. Chem. Phys. 71, 1007 (1979).

[19] This condition is not restrictive since in $1 \mathrm{D}$ we can use the non-degeneration theorem [L. D. Landau and E. M. Lifshitz, Quantum Mechanics: Non Relativistic Theory (Pergamon, Oxford 1959), p.57] to show that all eigenfunctions vanishing at infinity are real.

[20] P. D. Kirkman and J. B. Pendry, J. Phys. C 17, 4327 (1984).

[21] N. H. March and M. P. Tosi, Proc. R. Soc. Lond. A 330, 373 (1972).

[22] G. Grosso and G. Pastori Parravicini, Solid State Physics (Academic, London 2000) p. 191.

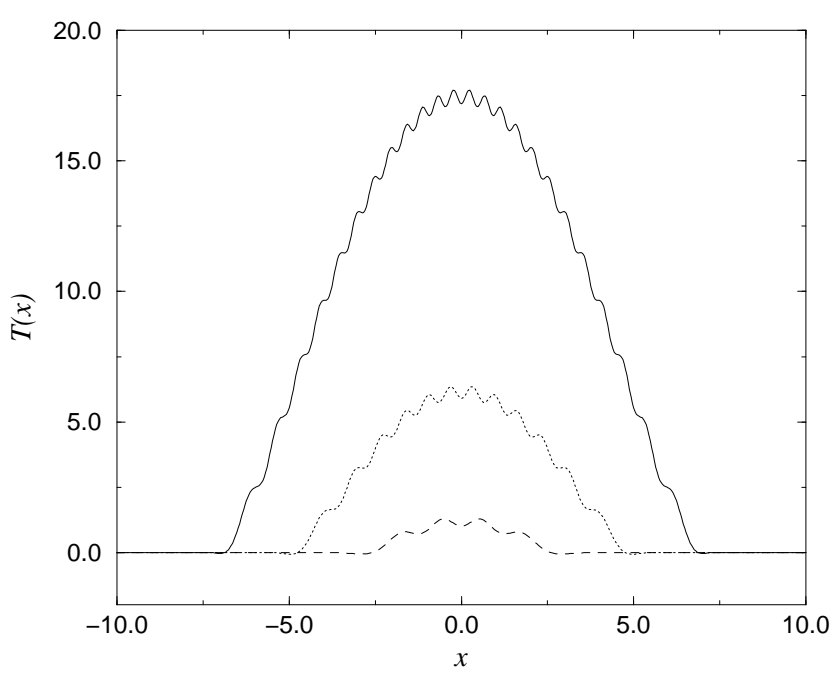

(a)

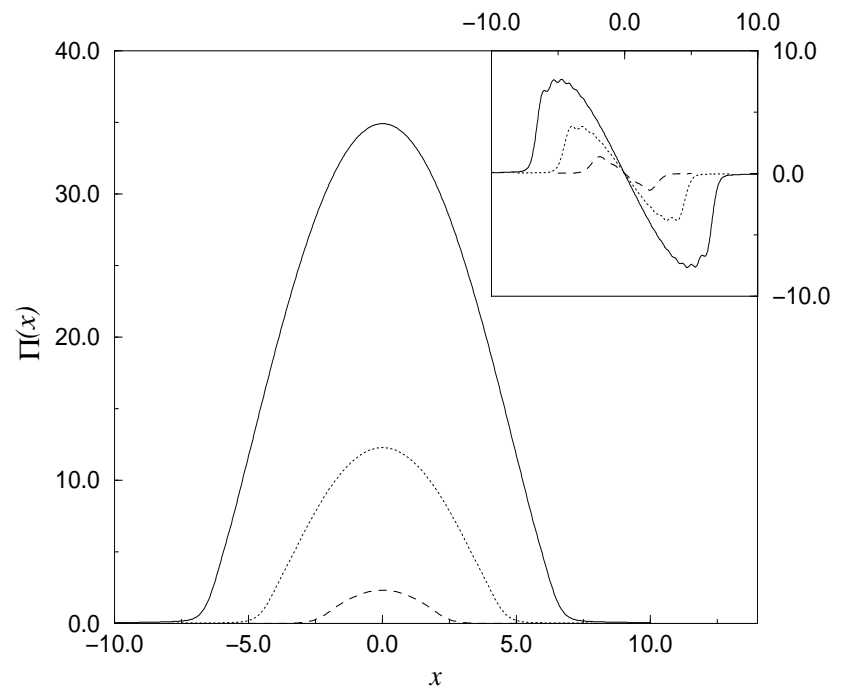

(b)

FIG. 1. Exact kinetic energy density $T(x)$ (a) and momentum flux density $\Pi(x)$ (b), in units of $\hbar \omega / a_{h o}$ as functions of the spatial coordinate $x$ (in units of $a_{h o}=\sqrt{\hbar / m \omega}$ ) for $N=4$ fermions (dashed line), 12 fermions (dotted line) and 24 fermions (solid line) in a 1D harmonic potential. The inset in (b) shows the derivative $d \Pi(x) / d x$. 


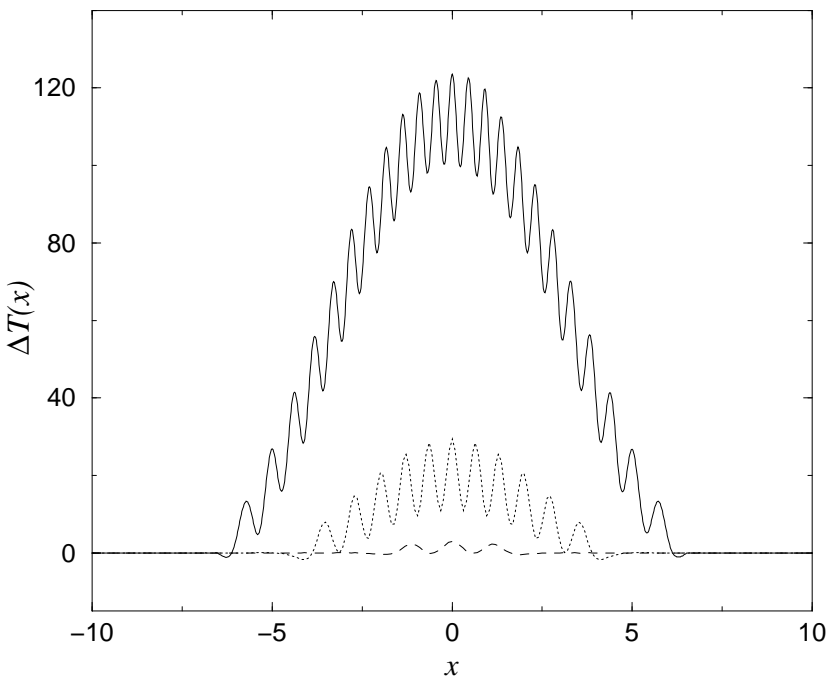

(a)

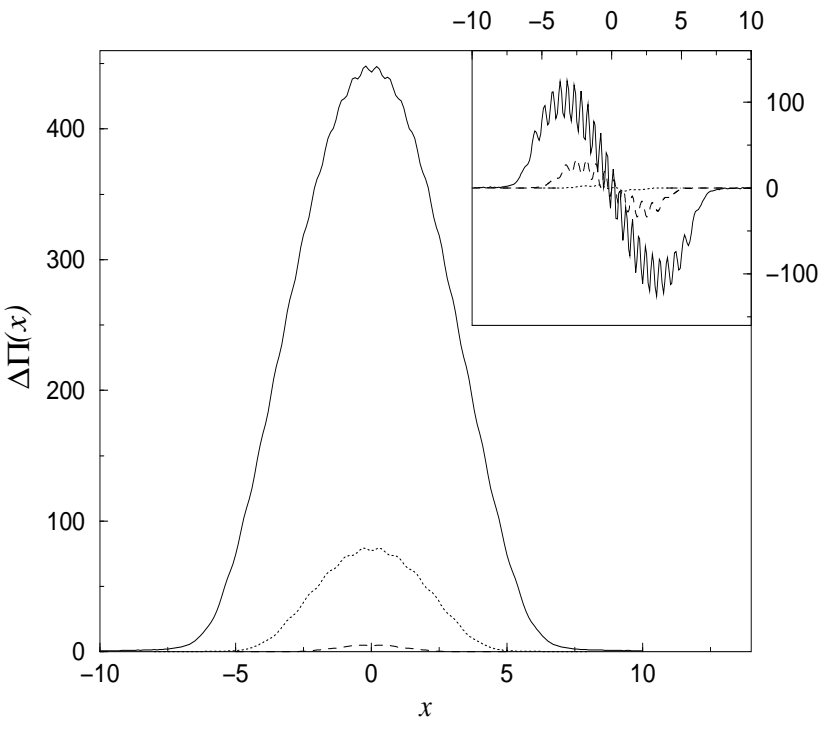

(b)

FIG. 2. Quantal fluctuations of the kinetic energy density (a) and of the momentum flux density (b) (in units of $\left.(\hbar \omega)^{2} / a_{h o}\right)$, as functions of the spatial coordinate $x$ (in units of $a_{h o}$ ). The numbers of particles and the symbols are the same as in Fig. 1. The inset in (b) shows the first derivative $d \Delta \Pi(x) / d x$.

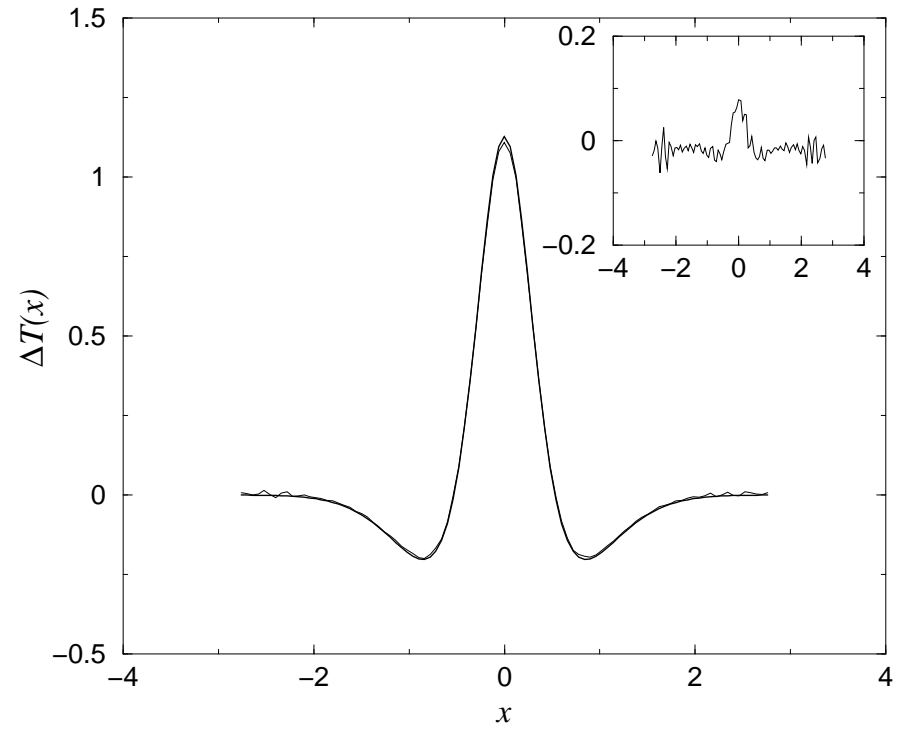

FIG. 3. Quantal fluctuations $\Delta T(x)$ of the kinetic energy density for a system of two fermions evaluated numerically by the Green's function method (dotted line), compared with the result of an analytic calculation (continuous line). The difference between the two curves is shown in the inset. The units are the same as in Fig. 2 . 


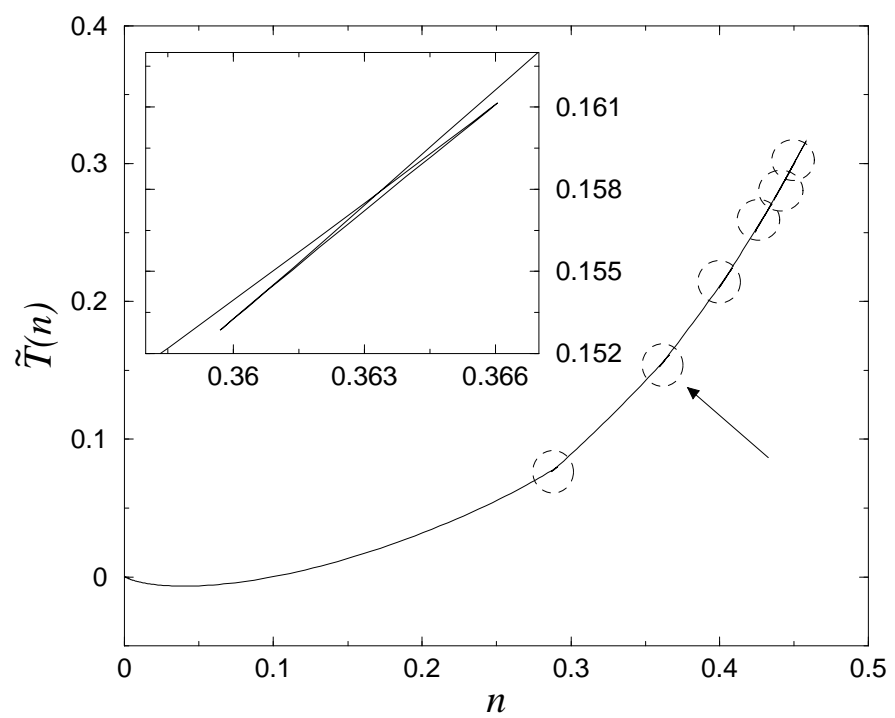

FIG. 4. Local kinetic energy density (in units of $N^{-3 / 2} \hbar \omega / a_{h o}$ ) as a function of the particle density (in units of $N^{-1 / 2} a_{h o}^{-1}$ ) for $N=12$. The circles indicate the regions in which the function is multi-valued. The inset shows an enlargement of one of these regions, indicated by the arrow. The function $\tilde{T}(n)$ is multi-valued around all local maxima of the density profile.

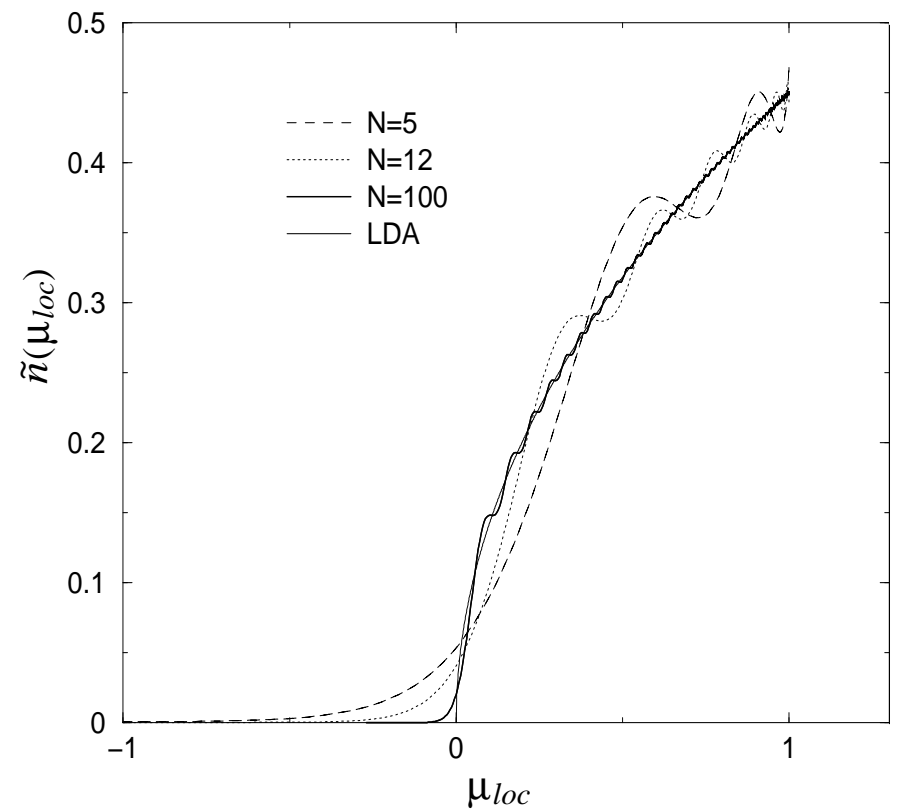

FIG. 5. Particle density $\tilde{n}$ (in units of $N^{-1 / 2} a_{h o}^{-1}$ ) as a function of $\mu_{l o c}$ (in units of $\hbar \omega / N$ ) for various values of $N$ as compared with the LDA profile. 